\title{
Preeclampsia y eclampsia en pacientes atendidas en el área de emergencia del Hospital Verdi Cevallos Balda julio 2016 - junio del 2017
}

\section{Preeclampsia and eclampsia in patients treated in the emergency area of the Hospital Verdi Cevallos Balda July 2016 - June 2017}

\section{Pré-eclâmpsia e eclampsia em pacientes tratados na área de emergência do Hospital Verdi Cevallos Balda julho 2016 - junho de 2017}

\author{
Carmen A. Condo-Baque ${ }^{\mathrm{I}}$ \\ carmcooar5@hotmail.com \\ Gema M. Barreto-Pincay II \\ gemabar-pin@gmail.com \\ Grace M. Montaño-Parrales III \\ grace-montano2@hotmail.com
}

Leonardo X. Borbor-Sánchez ${ }^{\mathrm{IV}}$ leonardo.borbor@gmail.com

Gabriela L. Manrique-Regalado ${ }^{\mathrm{V}}$ gabriela.manriqu5@hotmail.com

Alison J. García-Sigcha VI alison.garci@gmail.com

Recibido: 27 de febrero de 2018 * Corregido: 18 de mayo de 2018 * Aceptado: 15 de julio de 2018

\footnotetext{
I. Responsable de Promoción de la Salud e Igualdad Distrito 13D09 Pajan, Ecuador.

II. Médico - Residente Medicina Interna Clínica Santa Margarita, Portoviejo, Ecuador.

III. Lic. Enfermería “Centro de Salud Jipijapa” Distrito 13D03, Jipijapa, Puerto López, Ecuador.

IV. Médico Hospital Israel Quintero 13D09 Pajan, Ecuador.

V. Lic. Enfermería Centro de Salud Jipijapa Distrito 13D03, Jipijapa, Puerto López, Ecuador.

VI. Obstétrico, Hospital Rodríguez Zambrano Centro, Manta, Ecuador.
} 


\section{Resumen}

La preeclampsia severa es una de las complicaciones más frecuentes del embarazo y ha contribuido a la morbimortalidad tanto materna como neonatal, del 5 a $20 \%$ de los embarazos pueden complicarse con Preeclampsia y Eclampsia que lleva a un aumento significativo de la morbimortalidad materna y perinatal. El objetivo principal de la investigación es evaluar el Manejo de preeclampsia/eclampsia en el área de emergencia del Hospital Verdi Cevallos Balda identificando factores de riesgos personales y familiares. El estudio es prospectivo, descriptivo con un universo constituido por 3400 embarazadas que ingresaron al área ginecológica desde julio 2016 a junio 2017, el trabajo se efectuó en base a las estadísticas del Hospital. De acuerdo a los resultados entre 3400 embarazadas se reportaron 125 casos de Preeclampsia/eclampsia en mujeres de edades comprendidas entre 2130 años, ama de casa, de estado civil unión libre, de zona urbana, de instrucción primaria y que se realizaron pocos controles prenatales, el grado de preeclampsia más presentada fue leve entre las 37 a 46 semanas de gestación, con signos de hipertensión arterial, edema, cefalea, el manejo de la patología se lo realizó con sulfato de magnesio, en su mayoría los embarazos terminaron con cesárea y niños prematuros. El cumplimiento de normas se daba parcialmente ya que por el ingreso de nuevo personal desconocían las pautas a seguir. Se recomienda cada vez que ingrese nuevo personal se los capacite y actualicen sobre las normas, utilizar referencia y contrarreferencia, mantener un lazo estrecho entre las Unidades Operativas y enfocarse en captar lo más temprano a la embarazada, educarlas sobre lo importante que es realizarse los controles rutinarios.

Palabras claves: Preeclampsia, eclampsia, embarazo, protocolo, programa, prevención. 


\begin{abstract}
Severe preeclampsia is one of the most frequent complications of pregnancy and has contributed to both maternal and neonatal morbidity and mortality, from 5 to $20 \%$ of pregnancies can be complicated by preeclampsia and eclampsia leading to a significant increase in maternal and perinatal morbidity and mortality. The main objective of the research is to evaluate the management of preeclampsia / eclampsia in the emergency area of the Verdi Cevallos Balda Hospital, identifying personal and family risk factors. The study is prospective, descriptive with a universe constituted by 3400 pregnant women who entered the gynecological area from July 2016 to June 2017, the work was carried out based on the statistics of the Hospital. According to the results among 3400 pregnant women, 125 cases of pre-eclampsia / eclampsia were reported in women aged between 21 and 30 years, housewife, civil union, urban area, primary education and few prenatal check-ups were performed., the degree of preeclampsia most presented was mild between 37 to 46 weeks of gestation, with signs of hypertension, edema, headache, the management of the pathology was performed with magnesium sulfate, most pregnancies ended with caesarean section and premature children. Compliance with standards was partially due to the fact that new personnel were unaware of the guidelines to follow. It is recommended each time new personnel enter to train and update on the rules, use reference and counter-reference, maintain a close link between the Operating Units and focus on capturing the pregnant woman as early as possible, educate them about the importance of carrying out the controls routine.
\end{abstract}

Key words: Preeclampsia, eclampsia, pregnancy, protocol, program, prevention.

\title{
Introducción.
}

289 Vol. 4, núm. 3, julio 2018, pp. 278-293

Carmen A. Condo-Baque; Gema M. Barreto-Pincay; Grace M. Montaño-Parrales; Leonardo X.

Borbor-Sánchez; Gabriela L. Manrique-Regalado; Alison J. García-Sigcha 
La preeclampsia es un trastorno hipertensivo inducido por el embarazo que se manifiesta clínicamente después de las 20 semanas de gestación; la falta de un manejo oportuno, conduce a eclampsia, sin embargo, la causa sigue desconocida y se asocia a problemas de salud maternaperinatal importantes (1). La preeclampsia y eclampsia se manifiestan clínicamente con todo un espectro de síntomas clínicos por la misma condición, están presentes principalmente la hipertensión y proteinuria, además de edema, cuando se presentan convulsiones, además de estos síntomas, se diagnostica como eclampsia, algunas embrazadas desarrollan a su vez el Síndrome Hellp, caracterizado por alteraciones bioquímicas que evidencian hemólisis, elevación de enzimas hepáticas y plaquetopenia (2).

La preeclampsia constituye un gran problema dentro del área de salud, en el aspecto médico las complicaciones que se presentan pueden comprometer la vida de la paciente como para el producto o dejando secuelas importante, en el aspecto social interviene la parte psicológica por la pérdida del bebé, por un prematuro con problema o por una madre condenada a tomar antihipertensivos de por vida, y en el caso de los familiares por la pérdida de la madre e hijo, también tienen repercusión en el aspecto económico por el mantenimiento de una larga espera ante una mujer con un prematuro en termocuna. Todo esto hace que pongamos atención ante esta grave enfermedad por las repercusiones mencionadas.

La preeclampsia incide significativamente en las tasas de morbilidad y mortalidad materna perinatal a nivel mundial. Sin embargo, el impacto de la enfermedad es más severo en países en desarrollo, donde otras causas también frecuentes, ocasionan mortalidad materna (por ejemplo, hemorragia y sepsis) (3).

\footnotetext{
281 Vol. 4, núm. 3, julio 2018, pp. 278-293 
La Organización Mundial de la Salud (OMS) estima que la incidencia de preeclampsia es siete veces mayor en los países en desarrollo que en los desarrollados $(2,8 \%$ y $0,4 \%$ de los nacidos vivos respectivamente). La incidencia de eclampsia en los países desarrollados de Norteamérica y Europa es similar y se estima alrededor de 5 a 7 casos por cada 10.000 partos, mientras que en países en desarrollo es variable, oscilando entre 1 caso por cada 100 embarazos a 1 por cada 1.700 embarazos. Las tasas de los países africanos como Sudáfrica, Egipto, Tanzania y Etiopía varían de $1,8 \%$ a $7,1 \%$ y en Nigeria, la prevalencia oscila entre $2 \%$ a $16,7 \%(4)$.

En Latinoamérica, la morbilidad perinatal es de 8 al $45 \%$ y la mortalidad del 1 al 33\%, afectando al $40 \%$ de las mujeres con enfermedad renal crónica o trastornos vasculares (4). Primordialmente la alteración de la preeclampsia y eclampsia constituye una de las cuatro causas principales de mortalidad después de las hemorragias, abortos e infecciones, tanto para la madre como para su hijo. Esta enfermedad por tanto, ofrece una oportunidad importante de salvar vidas si se pueden prevenir las formas graves o hacer una detección temprana. Los fenómenos hipertensivos asociados al embarazo en particular la preeclampsia y eclampsia constituyen en muchos países subdesarrollados una de las principales causa de morbimortalidad materna-fetal e incrementando así el índice de cesáreas.

En Ecuador existe un índice del $21.1 \%$ de muertes por cada cien mil nacidos vivos, incluyendo muertes maternas por causas obstétricas ocurridas durante el periodo del embarazo, parto o post parto donde preeclampsia y eclampsia son la tercera causa de muerte materna alcanzando el $30 \%$ de los casos, hasta la actualidad el número de muertes maternas han logrado una leve reducción, sin embargo es necesario que se desarrollen mecanismos para disminuir en la totalidad este problema de salud (5).

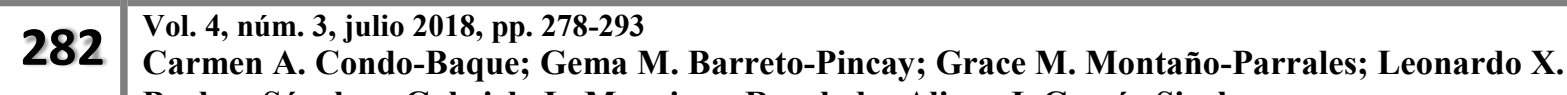
Borbor-Sánchez; Gabriela L. Manrique-Regalado; Alison J. García-Sigcha
} 
Los problemas con el manejo de la hipertensión en el embarazo se encuentran no solo en su etiopatogenia desconocida, sino también en las variaciones, en sus definiciones, medidas y clasificaciones utilizadas para categorizar la hipertensión en las embarazadas. La preeclampsia usualmente se ha considerado como una causa relevante de morbilidad neonatal, ya que genera gran parte de los nacimientos que se producen antes del término de la gestación o por el impacto negativo sobre el peso neonatal, que puede contribuir a su ingreso en neonatología. De hecho grandes estudios de cohorte han mostrado que todos los trastornos hipertensivos del embarazo implican mayor riesgo para bajo peso y para macrosomías (6).

Los resultados maternos fetales en pacientes con preeclampsia severa dependen de la edad gestacional en que se desarrolla la preeclampsia, la severidad de las enfermedades y la multiparidad. El manejo de la paciente constituye un reto en la actualidad para muchos colegas ya que el curso clínico de la misma se caracteriza por un deterioro progresivo de la salud de la madre y del feto y se asocian a un incremento considerable de la asfixia y muerte perinatal que ninguno de nosotros desearía tener.

Los retrasos en la prestación de atención médica, también contribuyen a la mortalidad materna por preeclampsia y eclampsia, debido a la falta de atención medica que necesitan antes, durante y después del parto, particularmente en situaciones de emergencia, incluso las actitudes y calidad de atención por los médicos a poblaciones marginadas también se identifican como barreras, esto se agrava por la falta de personal capacitado, equipos y suministros. Por ejemplo, a pesar de que la eficacia del sulfato de magnesio ha sido documentada por varios investigadores, los estudios han demostrado que el sulfato de magnesio no se administra rutinariamente, y su uso se limita a los

\footnotetext{
283 Vol. 4, núm. 3, julio 2018, pp. 278-293

Carmen A. Condo-Baque; Gema M. Barreto-Pincay; Grace M. Montaño-Parrales; Leonardo X. Borbor-Sánchez; Gabriela L. Manrique-Regalado; Alison J. García-Sigcha
} 
hospitales de enseñanza (7). La falta de disponibilidad de los medicamentos, personal de salud apropiados necesarios para su administración y costo son los obstáculos que suelen plantearse.

Los factores sociales influyen en la preeclampsia con $27 \%$ de las muertes maternas y es importante aumentar la conciencia en las mujeres sobre la necesidad de recibir atención de emergencia, si surgen complicaciones durante el parto, para asegurar la intervención médica rápida, eficaz e incrementar la probabilidad de éxito terapéutico (8) (9). Toda la comunidad debe reconocer los signos, desarrollar planes en situaciones de emergencia e incluir el traslado a hospitales, porque la reducción del riesgo de muerte se vuelve más difícil cuando se han desarrollado complicaciones.

Analizando el problema se pretende que con el presente estudio se realice acciones educativas a nivel del área de emergencia para que entre en vigencia el uso de las normas y protocolos del componente normativo materno emitido por el Ministerio de Salud Pública y así disminuir los casos de eclampsia en las gestantes atendidas.

\section{Material y métodos.}

El estudio se llevó a cabo en el área de Emergencia Ginecología del Hospital Verdi Cevallos Balda, en esta sala se atienden todos aquellos embarazos patológicos independientes de la edad gestacional con el objetivo principal es valorar el cumplimiento de los Protocolos de Atención de Preeclampsia-Eclampsia en el Hospital Verdi Cevallos Balda durante el periodo de julio del 2016 a junio del 2017

Hipótesis: Aplicando un Protocolo de Atención Médica a pacientes con Preeclampsia y Eclampsia se disminuirá la morbi- mortalidad.

\footnotetext{
284 Vol. 4, núm. 3, julio 2018, pp. 278-293 
Variables: Independiente (Paciente con Preeclampsia y Eclampsia), Dependiente (Propuesta de Mejoras) y variable intervinientes (Prevalencia, Grado de Preeclampsia, Características Gineco Obstétricas)

El diseño de estudio es descriptivo, retrospectivo y transversal, el universo estuvo constituido por todas las embarazadas 3400 que ingresaron al área de emergencia y la muestra son todas las pacientes que ingresan con diagnóstico de Preeclampsia- Eclampsia, 125 casos, como criterio de inclusión tenemos: cumplir el requisito de la definición de caso (Preeclampsia/Eclampsia), tener embarazo mayor de 20 semanas de gestación y aceptar participar en el estudio. Los criterios de exclusión son todas las pacientes con diagnóstico clínico de preeclampsia que no tenían completos sus exámenes de laboratorio.

Para la recolección de la información se utilizó un cuestionario, a las variables categóricas se les calculó proporciones. Las variables continuas fueron categorizadas para realizar el cálculo de proporciones. Los datos fueron procesados y analizados en el paquete estadístico Epi-Info y sus resultados están presentados en las tablas.

\section{Resultados.}

El presente estudio se realizó con 3400 mujeres embarazadas que ingresaron al área de ginecología de las cuales 125 casos resultaron pre-eclámpticas y eclámpticas. Se ha observado que la preeclampsia afecta entre el $2-10 \%$ de los embarazos, en este caso de 3400 pacientes que ingresaron a emergencia al área de ginecología 125 mujeres experimentaron Preeclampsia/Eclampsia correspondiéndola prevalencia al 3,6\% estudios, que va de la mano con estudios realizados.

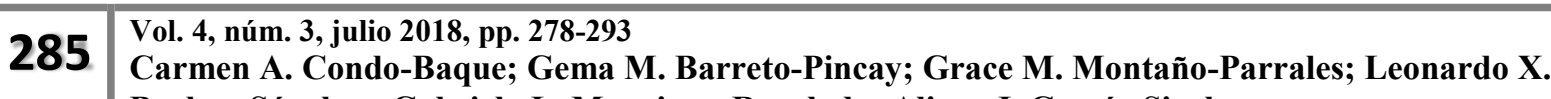
Borbor-Sánchez; Gabriela L. Manrique-Regalado; Alison J. García-Sigcha
} 
Del total de pacientes ingresadas en el servicio de Emergencia del Hospital Verdi Cevallos Balda de la Ciudad de Portoviejo en el período de estudio, con las siguientes características generales:

- Edades: El 24\% tenían un rango de edad entre 15 y 20 años, el $60 \%$ está entre 21 y 30 años y con un porcentaje del 13\% en edades comprendidas entre 31 y 43 años.

- Ocupación: El 73\% se desempeña como ama de casa, el 12\% como doméstica, el 9\% son estudiantes y el $6 \%$ son profesionales.

- Estado Civil: El 72\% de la población atendida es de unión libre, el 18\% son casadas y el $10 \%$ solteras.

- Procedencia: Urbano corresponde con el $62 \%$ y el $38 \%$ de la población estudiada habita en zona rural.

- Escolaridad: El 28\% son analfabetas, el 53\% tienen un grado de instrucción primaria, el $13 \%$ secundaria y el $6 \%$ tienen estudio superior.

En cuanto al grado de severidad de la Preeclampsia/Eclampsia se presentaron 81 casos de Preeclampsia leve correspondiendo al 65\%, 15 con preeclampsia moderada que representa el $12 \%$, 21 casos de preeclampsia severa con el 17\% y 8 casos de pacientes que presentaron eclampsia que representa el $6 \%$.

Según la edad gestacional encontradas en el estudio encontramos a 21 pacientes entre las semanas 20-27 semanas de gestación que corresponde al 17\%, el 37\% a las pacientes entre las 28 a 36.6 semanas, el $41 \%$ que representa 51 usuarias entre las 37 y 41.6 semanas y el $5 \%$ en las mayores de 42 semanas. 
Según el número de embarazos de las pacientes atendidas encontramos a 80 primigesta que representan el $64 \%$ y 45 multigestas que corresponde al $36 \%$.

En lo que respecta a las características gineco - obstétricas donde se verifico el número de controles prenatal se encontró un total de 15 pacientes sin control que corresponde al $12 \%$ que se presentaron entre zona urbana y rural, 70 casos con $1-2$ controles que representa al $56 \%$ y 40 pacientes con el $32 \%$ de 3 a 5 controles que por normas de Ministerio de Salud deberían realizarse 5 controles prenatales como mínimo.

En el estudio realizado se indago los factores de riesgo de la población en estudio donde la mayoría presentaron como factor de riesgo tanto personal como familiar la Hipertensión Arterial encontrando a 83 gestantes que representa el $66 \%$, seguidas de obesidad con el $31 \%$ que corresponde a 30 usuarias y diabetes con 30 pacientes que indica el $24 \%$.

De acuerdo a la sintomatología presentada el $100 \%$ experimento aumento de la presión arterial, el $89 \%$ presento cefalea, el $65 \%$ aumento de peso, el $45 \%$ presento edema, el $20 \%$ refirió alteración visual, el $17 \%$ epigastralgia, el $9 \%$ presento alteración auditiva y el $5 \%$ presentó convulsiones.

Según los exámenes del laboratorio, la creatinina mayor de $1.2 \mathrm{mg} / \mathrm{dl}$ se presentó en el $3 \%$ de las pacientes con preeclampsia/eclampsia. El ácido úrico mayor de $5.5 \mathrm{mg} / \mathrm{dl}$ se presentó en el 17\% de la pacientes, la proteinuria en orina al azar fue del $28 \%$ y la proteinuria de 24 horas mayor de $5 \mathrm{~g}$ realizadas corresponde al $66 \%$ y el $8 \%$ con trastorno hepático.

Al estudiar el tratamiento utilizado en las pacientes se encontró que en los tipos de preeclampsia y eclampsia no se cumplen a cabalidad con las normas implementadas por el

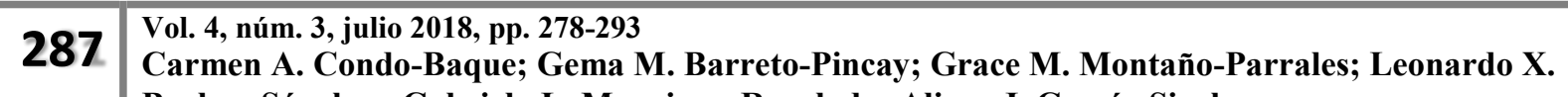
Borbor-Sánchez; Gabriela L. Manrique-Regalado; Alison J. García-Sigcha
} 
Ministerio de Salud Pública. En la preeclampsia no se prescribió hidralazina que el Hospital no contaba con este medicamento en la actualidad ya constan con este fármaco por vía Intravenosa (IV). Se indicó sulfato de magnesio en bolos a 34 pacientes con preeclampsia (27\%) y a 56 con dosis de mantenimiento (45\%), se manejó con Nifedipina al 55\% de las pacientes y reposo al 25\% de las pacientes.

La terminación del embarazo de las pacientes con preeclampsia fueron 86 pacientes que culminaron su embarazo en el hospital, 75 se sometieron a cesárea correspondiendo al $60 \%$ y 11 terminaron en parto que corresponde al $9 \%$, donde el $5 \%$ fue parto prematuro y el $4 \%$ el parto fue a término, existió el $20 \%$ que fueron enviadas con tratamiento ambulatorio y el $12 \%$ referidas a una unidad de mayor complejidad.

Se presentaron en las pacientes diagnosticadas con preeclampsia y eclampsia complicaciones o repercusiones como sufrimiento fetal en 8 de los partos que representa el $6 \%$, prematurez en 6 de los casos con el 5\%, óbito fetal en el 4\% de los casos y síndrome de Hellp en 3 pacientes con el 2\%.

Previa evaluación del programa concluimos que se había mejorado con las normas y protocolos pero de manera parcial encontrando un $62 \%$ de cumplimiento de acuerdo a lo establecido por el Ministerio de Salud Pública se propuso capacitaciones frecuentes sobre el manejo de preeclampsia y eclampsia para así disminuir la morbi- mortalidad materna y neonatal.

En la evaluación de las historias clínicas de las pacientes en estudio constatamos que en el ítem de anamnesis todas estaban completa y correctamente llenadas, al igual que le examen físico: reflejos, edema, actividad uterina, evaluación materna. En Los exámenes de laboratorio constan de 
evaluación hematológica, renal, metabólica, Los Estudios por imágenes maternas ecografía, EKG, eco cardiograma, fondo de ojo, e imágenes fetales flujometria Doppler y monitoreo fetal electrónico.

\section{Discusión.}

La Prevalencia en cuanto a edad representa el $60 \%$ entre 21 y 30 años, el $73 \%$ se desempeña como Ama de casa, de estado civil Unión libre con 72\%, de procedencia urbana que corresponde al $62 \%$, y escolaridad primaria con $53 \%$ ya que no se realizan los suficientes controles prenatales y no conocen acerca de los problemas que se pueden presentar en un embarazo sin controles.

Se ha observado que la preeclampsia afecta entre el $2-10 \%$ de los embarazos y se presenta principalmente en las pacientes con edad materna menor de 20 años y mayor a los 35 años, con estado socioeconómico bajo, en el estudio se observa que el grupo etareos entre 21 y 30 años presentó el mayor porcentaje de pacientes con preeclampsia. Según la procedencia el $62 \%$ son del área urbana y el $38 \%$ son del área rural lo que puede ser debido a la poca accesibilidad de las pacientes a los centros de salud y al sistema de referencia a los diferentes centros hospitalarios.

Los datos demuestran que se presentan mayores pacientes con Preeclampsia leve correspondiendo al 65\% lo que indica que basando en las normas y protocolos del MSP si podremos disminuir los casos de morbi-mortalidad de esta patología frecuente seguida por la Preeclampsia severa, moderada y 8 casos de eclampsia.

Dentro de la edad gestacional las semanas en las que más se ha presentado la Preeclampsia/Eclampsia son: 37 a 41 semanas correspondiendo al el $41 \%$ seguidos muy de cerca de 28 a 36.6 semanas representando el $37 \%$, se trataba de mantener un estado no grave para evitar la cesárea y a consecuencia un bebe prematuro que necesite de maduración pulmonar con surfactante.

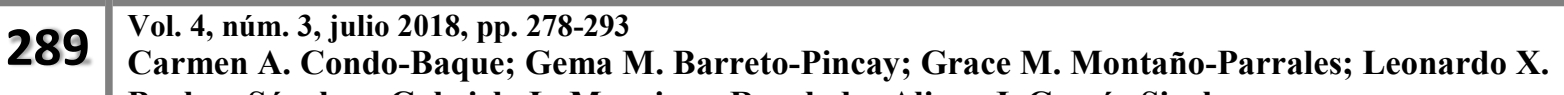
Borbor-Sánchez; Gabriela L. Manrique-Regalado; Alison J. García-Sigcha
} 
La fuente estadística demuestra que la Preeclampsia/Eclampsia se presenta en Primigestas con el $64 \%$, lo que indica que el mayor caso se presentaba por falta de controles prenatales más aún con antecedentes personales y familiares. En el estudio realizado se constató que en las pacientes que se presentó esta patología fueron quienes se habían realizado entre 1 y 2 controles prenatales, ya que la falta de controles y factores de riesgo son indicadores de la presencia de la preeclampsia.

Entre los factores de riesgo que más se presentaron fueron hipertensión, seguidos de los antecedentes familiares de hipertensión, obesidad y diabetes, se puede presentar en un $7 \%$ las pacientes que experimentaron una preeclampsia anterior. Además, es necesario mencionar que varias pacientes presentaron más de un factor de riesgo.

En la mayoría de los casos la sintomatología es similar a los estudios realizados presentando el aumento de la presión arterial en el total de los casos representando el 100\% seguido por cefalea $89 \%$, aumento progresivo del peso corporal $65 \%$, tomando a consideración que se presentaron convulsiones. En la bibliografía consultada el síntoma principal de la preeclampsia a más del incremento de la presión arterial es la cefalea en un $89 \%$ seguido aumento de peso, edema, alteraciones visuales, dolor epigástrico y convulsiones en el caso de la eclampsia.

El flujo sanguíneo renal y el índice de filtración glomerular descienden en la preeclampsia. Considerando que una reducción del IFG del 50\% duplica la creatinina sérica, un aumento de la misma representa una disminución del filtrado glomerular del 50\%. En la preeclampsia aumenta el ácido úrico plasmático frecuentemente antes de una elevación mesurable de la creatinina, indicando un menor daño renal (10). 
Siendo la hiperuricemia un marcador valioso para diferenciar la preeclampsia de todas las demás causas de hipertensión del embarazo. La hiperuricemia se correlaciona bien con la severidad clínica de la preeclampsia, con la lesión histológica detectada en la biopsia renal y la supervivencia fetal (11). En el estudio se vio alterada la creatinina en el 3\% de las pacientes con preeclampsia, lo que indica que a mayor elevación de la presión arterial se presentó mayor alteración de la función renal. Mayor número de pacientes presentaron aumento del ácido úrico en relación a la creatinina relacionándose con la severidad clínica de dicha patología (12).

En las normas lo indicado en la preeclampsia es la hidralazina pero el Hospital no contaba con este medicamento, en la actualidad ya constan con este fármaco por vía intravenosa (IV), se inició con sulfato de magnesio en bolos a 34 pacientes con preeclampsia que corresponde al $27 \%$ con y a 56 con dosis de mantenimiento que representa el 45\%, se manejó con Nifedipina al 55\% de las pacientes y reposo al $25 \%$ de las pacientes en sala de alto riesgo, el reposo es un factor muy importante ya que disminuye el stress y por lo tanto disminuye la presión arterial.

El tratamiento médico de las pacientes con preeclampsia consiste en el uso de Hidralazina que es el antihipertensivo ideal porque que reduce la presión arterial de manera gradual en nuestro medio se usó el sulfato de magnesio como fármaco de entrada y de mantenimiento y preventivo de convulsiones para el manejo de las alteraciones del sistema nervioso (13). En las pacientes con preeclampsia leve la atención satisfactoria suele consistir en reposo y observación la que puede llevarse a cabo en casa o en el hospital.

En la mayoría de las pacientes se indica reposo que minimiza la actividad vasopresora, aumenta al máximo el flujo sanguíneo útero placentario, genera presiones más bajas y promueve la diuresis. En el presente estudio a las pacientes con preeclampsia leve se les indicó tratamiento

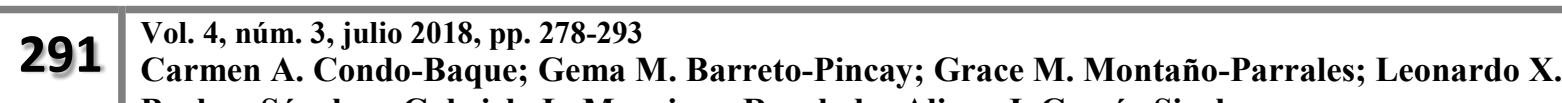
Borbor-Sánchez; Gabriela L. Manrique-Regalado; Alison J. García-Sigcha
} 
antihipertensivo, prescribiendo al $10 \%$ de las pacientes hasta dos fármacos para controlar la presión y menos de un tercio de las pacientes se le indicó sulfato de magnesio.

La mayoría de las pacientes independiente del tipo de preeclampsia terminaron ya sea por parto o cesárea debido a que eran productos de término y un pequeño porcentaje fue manejado ambulatoriamente las que no tenían alteraciones de laboratorio y la presión arterial diastólica era menor de $90 \mathrm{mmHg}$ y estaban cerca de un centro de salud cumpliéndose con lo indicado en la literatura.

En la finalización del estudio se evaluó sobre las medidas del término del embarazo expresando que la mayoría de los casos fueros cesárea con 75 pacientes que representa el $60 \%$, es sugestivo y peligroso que sometan a inducción del parto a una mujer con preeclampsia pero tampoco es justificación que aumente el índice de cesárea como se lo ha hecho hasta ahora, solo 5 usuarias que corresponde al $4 \%$ lograron llegar a término el parto.

Se ha reportado las diferentes complicaciones maternas y fetales entre ellas tenemos: Eclampsia en un 4\%, Presión arterial no controlada, óbito fetal y sufrimiento fetal agudo, lo que puede ser debido al sistema de referencia de los centros de salud hacia los hospitales o a las modificaciones de la conducta en el manejo de interrupción del embarazo, una vez estabilizada la paciente no se espera que ocurra otra descompensación.

Al estudiar el tratamiento utilizado en las pacientes se encontró que en los tipos de preeclampsia y eclampsia no se cumplen a cabalidad con las normas implementadas por el Ministerio de Salud Pública, sin embargo en la actualidad se ha mejorado por ello el cumplimiento 
radica en el $68 \%$ pero persiste el $32 \%$ cabe recalcar que la mejoría se demostrara con el uso continuo y las réplicas frecuentes del protocolo.

\section{Bibliografía.}

1. Vargas Hernandez V. Enfermedad hipertensiva durante el embarazo. Rev. Hosp. Jua. Mex. 2013 Marzo; VI(34).

2. López M. Preeclampsia-eclampsia: Un problema médicodiferido. Gac Méd Méx. 2011;(135).

3. World Health Organization. Global Program to Conquer Preeclampsia/ Eclampsia Washington: OMS; 2002.

4. Shah A, Fawole B, MÍmunya J. Resultado de parto por cesarea de la encuesta mundia de la OMS sobre la salud materna y perinatal en Africa. Intl J Gynec Obstet. 2012 Septiembre; 107(191).

5. Instituto Nacional de Estadistica y Censo. Los indices de mortalidad materna. [Online].; 2015 [cited 2016 enero 15.2 Available from: https://www.eluniverso.com/2015/10/08/infografia/5172098/indices-mortalidad-materna.

6. Gomez Gomez M, Danglot Banck C. El pronostico de los hijos de madres con preeclampsia. Mediagraphic Artemisa. 2014 Marzo - Abril; II(73).

7. Conde Agudelo A, Belizan J. Rish factors for pre-eclampsia in a large cohort of Latin American and Caribbean women. Brinnt Obstet Gynaecol. 2000; 107(75-83).

8. Kharb S. Serum markers in pre-eclampsia. Biomarkers. 2013; 14(395-400).

9. Duley L. Preeclampsia, eclampsia e Hipertension. Clinica evidencia. 2015; 2(14).

10. Vázquez-Rodríguez J, Rico-Trejo E. Papel del ácido úrico en la preeclampsia-eclampsia. Ginecol Obstet Mex. 2014 febrero; 79(5).

11. Packer C. Marcadores bioquimicos y parametros fisiologicos como indices para identificar pacientes con preclampsia. J. Hypertens. 2015 Junio; 45(23).

12. Trujillo M. MANEJO DE LAS PACIENTES CON PREECLAMPSIA EN EL SERVICIO DE ARO. Tesis doctoral. Nicaragua: UNIVERSIDAD NACIONAL AUTÓNOMA DE NICARAGUA, Facultad Ciencias Medicas; 2014.

13. Ministerio de Salud Publica del Ecuador. Componente Normativo Materno Ecuador: Consejo Nacional de Salud; 2008. 